\title{
The Effects of Neurofeedback Training on Memory Performance in Elderly Subjects
}

\author{
Gaël Lecomte, Jacques Juhel \\ Centre de Recherche en Psychologie, Cognition et Communication, \\ Université Européenne de Bretagne Rennes, France. \\ Email: jacques.juhel@univ-rennes2.fr

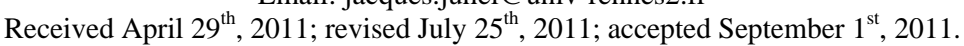

\begin{abstract}
Neurofeedback or electroencephalographic operant conditioning (EEG-OC) is an EEG biofeedback technique used to train individuals to control or modify their cortical activity through learned self-regulation. Initially used for treating a variety of pathologies, neurofeedback has been employed more recently to improve the physical or cognitive performance of human beings. The purpose of this study is to assess the hypothesis of the effect of neurofeedback (the 'awakened mind' model) on the memory performance of subjects aged over 65. 30 participants were shared equally between 3 groups: an experimental group that underwent 4 neurofeedback training sessions; a non-neurofeedback group trained at relaxation; and a 'waiting list' control group. Results showed that the members of the Neurofeedback group learned to increase the spectral power of the alpha frequency range as well as the alpha/thêta ratio, and that compared with the members of the two other groups, neurofeedback training resulted in a more pronounced decrease, albeit without any relation to changes in EEG activity and the level of stress and anxiety of participants undergoing such training. Yet contrary to expectations, no improvement of memory performance (differed recall of words and learning of lists of words) was observed. These mixed results, which suggest a wide range of applications, underline the need for a more systematic assessment of the potential applications of NFB training in elderly humans in order to be better able to specify the effects of the retained protocol on cognitive performance.
\end{abstract}

Keywords: Aging, Neurofeedback Training, Alpha Stimulation, Memory

\section{Introduction}

Neurofeedback training is based on the principle of operant conditioning and involves informing the subject in real time about the workings of their organism in order to incite them to modify their behaviour. The term "biofeedback" is used when the information provided concerns physiological parameters such as body temperature, breathing rhythm and heart rate. Biofeedback has been used for treating migraines (Nestoriuc, \& Martin, 2007), Raynaud's disease (Katsamanis Karavidas, Tsai, Yucha, McGrady, \& Lehrer, 2006) and urinary incontinence (Glazer \& Laine, 2006). Neurofeedback training (NFB) or electroencephalographic biofeedback (EEG) involves providing the subject in real time with information relating to the rhythmic cortical electrical activities that reflect the electrical activity of specific cortical areas and functions (Evans, \& Abarbanel, 1999; Masterpasqua, \& Healey, 2003). The aim of NFB is to enable the subject to become aware of particular patterns of cortical activity that we know or assume to be associated with a more optimal behaviour or state. The information returned to the subject in the course of an NFB session therefore pertains to the activity of one or several frequency bands (delta: 0 - $4 \mathrm{~Hz}$; thêta: $4-7 \mathrm{~Hz}$; lower and upper alpha: $7-12 \mathrm{~Hz}$; sensorimotor rhythms: 12 - $15 \mathrm{~Hz}$; beta: $15-20 \mathrm{~Hz}$; gamma: $30-80 \mathrm{~Hz}$ ). Feedback can be auditive and/or visual, more or less explicit (graphic or video games/animations), presented continuously or discontinuously, and can be based on fixed triggering thresholds or adaptive thresholds associated with absolute targetvalues (range of cortical waves) or relative target-values (for example, the proportion of global activity).

Neurofeedback has been used for treating epileptic patients (Sterman, \& Egner, 2006) and subjects suffering from attention deficit and hyperactivity disorders (Butnik, 2005), but also in the treatment of addictions (Scott, Kaiser, Othmer \& Sideroff, 2005), depression and anxiety (Hammond, 2005, 2006), and for monitoring the after-effects of neurological lesions (Bearden, Cassisi \& Pineda, 2003) and treating cases of chronic pain in elderly subjects (Middaugh \& Pawlick, 2002). Several studies have also focused on the effects of NFB on cognitive performance in healthy subjects (Budzynski, 1996; Egner \& Gruzelier, 2004; Gruzelier, Egner \& Vernon, 2006; Hanslmayer, Sauseng, Doppelmayr, Schabus \& Klimesch, 2005; Vernon, 2005; Vernon, Egner, Cooper, Compton, Neilands, Sheri \& Gruzelier, 2003).

The conceptualisation of NFB as an agent of cognitive change is essentially based on the correlations observed between certain EEG frequency bands and various aspects of information processing (Klimesch, 1999; Klimesch, Vogt \& Doppelmayer, 2000; Klimesch, Schack, \& Sauseng, 2005; Sauseng, \& Klimesch, 2008). For example, thêta activity appears to be related to working memory processes and episodic memory. It appears that lower alpha waves are largely associated with attentional processes, while upper alpha waves reflect recovery processes in semantic memory. Bêta waves associated with motor activity are also assumed to be involved in the activation of attentional processes. Finally, gamma activity may play a 'universal' role in sensory and cognitive processing (Basar, Basar-Eroglu, Karakas, \& Schürmann, 2000). The amplitude of lower frequency bands (delta and thêta) and upper frequency bands (gamma) tends to increase in line with cognitive effort while the amplitude of alpha and beta waves tends to decrease (Basar, 2004; Basar, Basar-Eroglu, Karakas, \& Schürmann, 2001). More recently, Sauseng, \& Klimesch (2008) have argued that phase synchronization (i.e. cohesion between corti- 
cal sites, between oscillations of differing frequency, with external stimuli) may be the underlying neural mechanism in certain cognitive processes. However, Sauseng and Klimesch remark that "we do not know if the association between physiological mechanisms and cognitive functions is correlational or causal $[\cdots]$ even if the results of several psychopharmacological and clinical studies suggest attributing a causal role to phase synchronization in information processing” (p. 1010).

The above remarks point to the application of NFB training protocols targeting frequency bands related to the cognitive functions that are deemed to require improved efficiency. Yet the complexity of the relations between EEG activity and cognition means that it is extremely difficult to clarify a priori the cognitive objectives of an inhibition or stimulation protocol of the activity of a given frequency band (thêta, alpha or bêta training). This clarification is even more problematic in the case of training protocols in which the participant must learn to amplify a level of activity (e.g. thêta) in conjunction with the inhibition of another (e.g. delta). Although results may sometimes be uncertain, observations of young adults and a number of findings drawn from studies of elderly subjects suggest several hypotheses.

Bauer (1976) was the first to study the effect of 4 NFB sessions (alpha stimulation) on short-term memory in young adults. The results showed an increase of alpha activity but did not point conclusively to an effect of NFB on memory performance. More recently, Vernon et al. (2003) studied the effects of two different NFB training modalities on the performance of young adults in a memory task involving semantic work (conceptual span paradigm) and in a visual attention task (continuous performance paradigm). The first training condition was a stimulation of thêta waves $(4-7 \mathrm{~Hz}$, in connection with working memory) and an inhibition of delta waves $(<4 \mathrm{~Hz}$, associated with sleep) and alpha waves (8 - $12 \mathrm{~Hz}$, associated with physical relaxation). The second training condition simultaneously included a stimulation of SMR waves $(12-15 \mathrm{~Hz}$, associated with attention) and an inhibition of bêta waves (18 - $22 \mathrm{~Hz}$, associated with problem-solving, and also sometimes anxiety disorders). Subjects took part in 8 individual sessions with feedback in the form of a video game. The observations drawn from the bêta-SMR condition chiefly highlighted an NFB learning process and a significant improvement of performance in the task involving semantic working memory and to a lesser extent in the attention task. The observations drawn from the stimulation condition of thêta waves and from the inhibition of alpha waves did not indicate any changes of EEG activity or of the level of cognitive performance. However, Hanslmayer et al. (2005) showed that alpha/thêta training (alpha stimulation, thêta inhibition) may result in an improvement of cognitive performance (mental rotation task) in participants capable of learning to increase the alpha/thêta ratio. It appears therefore that NFB training that simultaneously targets several objectives in relation to the frequencies that make up the initial profile of cortical activity and that simultaneously restitute information relating to several frequency bands (particularly thêta and alpha) may prove to be efficient, at least in 'responsive' individuals.

Although it has been clearly established that ageing involves a decrease of EEG activity (Obrist, 1954) and a decrease of the power of the alpha band (Markand, 1990), very few studies have so far focused on the impact of NFB on EEG activity and the cognitive performance of elderly subjects (Albert, Andrasik, Moore, \& Dunn, 1998; Angelakis, Stathopoulou, Frymiare, Green, Lubar, \& Kounios, 2007; Fernandez, Becerra, Roca,
Espino, Bahlke, Harmony, Fernandez-Bouza, Belmont, \& DiazComas, 2008). The only study in the area is by Angelakis et al. (2007), who target peak alpha frequency (PAF), which measures the discrete frequency of maximum amplitude in the alpha band. The PAF is known to be weaker in children and elderly individuals, and is also weaker in individuals suffering from Alzheimer's disease than it is in healthy subjects (Klimesch, Vogt \& Doppelmayer, 2000). The PAF is also correlated positively (independently of age) with the intellectual level of healthy individuals or of individuals suffering from neurological disorders (Angelakis, Lubar, \& Stathopoulou, 2004). Angelakis et al. (2007) administered over 30 NFB sessions to 6 individuals aged 70 to 78 . Three of the subjects underwent a training process aimed exclusively at increasing the PAF (experimental condition). Two other participants underwent an NFB protocol aimed at increasing the amplitude of alpha waves (8 $13 \mathrm{~Hz}$ ) (control condition 1). The sixth participant underwent a pseudo-NFB protocol (control condition 2: restitution based on the recorded EEG activity of another subject). Assessments of EEG activity at rest and during cognitive activities involving attentional control (number span, Stroop test, Raven's matrices, etc.) were conducted before and after the series of NFB sessions. Angelakis et al. (2007) observed an increase of PAF, which was particularly pronounced in frontal areas, in 2 of the 3 participants of the experimental condition and an increase of alpha activity in the 2 participants of the control condition. In parallel, the results of the cognitive assessments indicate that NFB PAF training improves the speed of information processing as well as the resistance to interference; training the amplitude of the alpha waves improves memory performance. These initial results are compatible with the hypothesis of a positive impact of NFB (stimulation of alpha waves) on the cognitive performance of elderly subjects. This effect could be explained by the facilitating role performed by the slower rhythms in the connections between different cortical areas (Gruzelier, Egner, \& Vernon, 2006).

The aim of this research was to examine these initial findings in the light of the observations of elderly subjects trained specifically to increase the power of the upper alpha frequency band $(10-12 \mathrm{~Hz})$ in comparison with the power of the thêta band $(4-7 \mathrm{~Hz}$ ) (Hanslmayer et al., 2005). The NFB training sessions undergone by participants in the experiment were expected to generate two chief observations: first of all, a change of EEG activity resulting in an increase of the alpha/thêta ratio; secondly, an improvement of cognitive performance, measured in this case in the area of short-term memory.

\section{Method}

\section{Participants}

Thirty subjects ( 23 women and 7 men aged 75.25 on average, age range: 65 to 85 ) took part in this study. The participants, who all lived at home, presented no known neurological or psychiatric antecedents or any known psychological disorder. None of the participants had undergone psychotropic treatment. Normality of performances in the Mini Mental State Examination (MMSE) was also required to minimize the probability of an inclusion of emerging degenerative pathologies.

\section{Procedure}

The experiment design and data collection procedure incorporated recent recommendations concerning the need to have EEG measurements in pre- and post-tests and the need to in- 
clude a non-contingent control group (Vernon, 2005). Participants were shared randomly between an experimental group, a control group and a test/retest group of equal sizes. On 4 separate occasions every week, the members of the experimental group (NFB) and of the control group (RELAX) were studied individually for approximately 1 hour. The content of each session varied according to the condition: a) experimental: 5minute preparation, 30-minute NFB training session, 5 minutes for removal of captors and recovery; or b) control: 5-minute warm-up, 30-minute dynamic relaxation session (simple yoga movements) and static relaxation (return to rest), 5-minute recovery. The session ended with a rapid self-assessment of the anxiety experienced by participants and of the stress generated by the situation ( 5 minutes) followed by a debriefing session lasting approximately 10 minutes. The assessment of cognitive performance was conducted before session 1 (20 minutes) and was repeated after session 4 . The third group (the "waiting list" group) was a test/retest group (TRT). The subjects placed in this condition took part in all the evaluations (first cognitive evaluation, self-assessment of stress and anxiety once a week and on 4 separate occasions, second cognitive evaluation) but did not benefit from any particular intervention.

\section{Psychological Assessment}

Cognitive performance in the area of memory was assessed by 2 of the 3 memory tests drawn from the Signoret Memory Battery (Signoret, 1996). The first test, known as the 'Recall' test (REC), involved a delayed recall of 6 images (after resolution of 6 arithmetic and verbal problems and a semantic fluency test). The second test, known as the 'learning' test (LEA), assessed the ability to memorize a list of 8 words immediately and the improvement of performance through repetition. The correlation between these tests was moderate $(\mathrm{r}=.409)$ on a sample of 50 elderly. Both tests were rated on a 12-point scale; the total number of points scored by participants could therefore amount potentially to 24 . The level of stress generated by the situation and the level of anxiety experienced during the session were evaluated using a non-graduated Visual Analogical Scale (0 - 10) with a recall of the preceding self-assessment. Both scores were then combined into one single score or 'tension level' (TENS).

\section{EEG Data Collection and Neurofeedback Protocol}

The collection of NFB data was conducted using the Pendant ${ }^{\circledR}$ EEG system and BioExplorer software for the purposes of recording and controlling/monitoring in real time. The analyses and return in the form of graphic animations and auditive signals correlated with cortical activity and the optimal profile of targeted activity were carried out using the BioReview sub-module provided by BioExplorer. Two pairs of captors were set up transversally: $\mathrm{C} 3-\mathrm{Cz} / \mathrm{Cz}-\mathrm{C} 4$, where $\mathrm{Cz}$ represents the reference points at the top of the skull; C3 and C4 represent the measurement sites in the middle of each hemisphere.

The training protocol used in this study targeted the cerebral activity of individuals who are expert practitioners of meditation (Cade, \& Coxhead, 1979). The practice of meditation aims to develop the ability to activate a conscious self-regulation of sensory and motor aspects of the physical body. When performed on a regular basis, this practice appears to have a positive impact on attention span (Brefczynski-Lewis, Lutz, Schaefer, Levinson \& Davidson, 2007) and on synchronization between cerebral areas in the gamma frequency bands $(30-70 \mathrm{~Hz})$ that correspond to the completion of metacognitive tasks in which self-regulation plays a key role (Lutz, Greischar, Rawlings, Ricard \& Davidson, 2004; von Stein, Chiang, \& Konig, 2000). The NFB protocol therefore aimed to harmonize the different ranges of cerebral waves with a view to tending towards an optimal reference model that describes the chief characteristics of the cerebral profile of experts in meditation (the so-called 'awakened mind' model; see Figure 1). It involved a combination of four pairs of filters in comparative mode (5 minutes for each), with one of these comparisons stimulating alpha waves in relation to thêta waves and another comparison inhibiting thêta waves in relation to slow bêta waves.

The stimulation process was conducted using a continuous sound signal (eyes closed) and a video animation on replay (eyes open) so long as it remained within an amplitude range enabling the subject to direct, for each of the generated ranges of cortical waves, the respective proportions of the different wave ranges towards the targeted waves. The sound feedback (continuous sound replay) and visual feedback (replay of video) were triggered whenever the conditions of the filters were satisfied simultaneously. The program automatically adjusted the triggering threshold according to the rate of success of the participant in order to avoid causing a sense of frustration or failure, while gradually increasing the level of demand of the triggering thresholds.

The relation between the spectral powers of the alpha and thêta bands measured during sessions 1 and 4 were computed as follows. The first three minutes of EEG data were collected at the beginning of session 1 and 4 and were then processed by normalizing the specific powers of the higher alpha frequency bands $(10-12 \mathrm{~Hz})$ and thêta frequency bands $(4-7 \mathrm{~Hz})$ and by relating them to the power of the signal for all frequencies between $2 \mathrm{~Hz}$ and $49 \mathrm{~Hz}$ while extracting the noise caused by electromagnetic parasites on either side of the band. 250 segments were used based on the Fast Fourier Transform algorithm, with a resolution of $.5 \mathrm{~Hz}$. The average alpha/thêta ratio for the channels assigned to $\mathrm{C} 3$ and $\mathrm{C} 4$ (with reference in $\mathrm{Cz}$ ) were then computed

\section{Statistical Analysis}

Since the NFB group included just 10 participants, the impact of NFB on EEG activity was assessed using the exact Wilcoxon test (unilateral test) by comparing the observations made in session 4 (spectral power of alpha and thêta frequency bands, relation between these spectral powers) with the observations drawn from session 1 . For every member of the NFB group, we also computed an index measuring changes in EEG activity in comparison with the average change observed in all

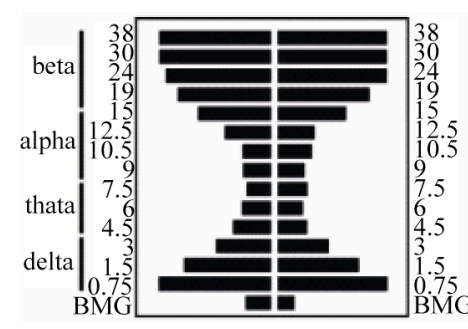

(a)

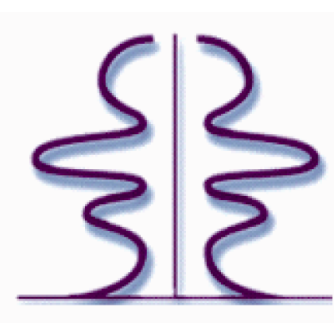

(b)
Figure 1.

a) Example of a pattern of electrical activity of the brain; b) Optimal targeted pattern ("Awakened Mind” model). 
the other participants within the group. The index we used was the Reliable Change Index (RCI), defined by Jacobson \& Truaux (Maassen, 2004), a statistical indicator of centered and reduced normal distribution.

The assessment of the impact of NFB on memory performance was conducted using a MANOVA with repeated measurements (2 dependent variables: REC and LEA; 1 betweensubjects factor with 3 modalities: NFB, RELAX and TRT; 1 within-subjects factor with 2 modalities: session 1 and session 4). The null hypothesis of evolution of memory performance identical to the one observed on average in members of the TRT group was tested in every member of the NFB group using the RCI. Finally we used an ANOVA with repeated measurements ( 1 between-subjects factor with 3 modalities: NFB, RELAX and TRT; 1 within-subjects factor with 4 modalities: sessions 1 to 4 ) to evaluate the evolution of tension level. The significance threshold retained for all the statistical analyses was fixed at .05 .

\section{Results}

We began by verifying that members of the experimental group (NFB), of the control group (RELAX) and of the test/ retest group (TRT) had the same average age, $\mathrm{F}(2.27)<1$, p $>.05$, and the same initial level of performance in the 'recall' and 'learning' tests, $\mathrm{F}(4.52)=.970, \mathrm{p}>.05$. Some scores in deficit by 8 (non deficit limit score $=9 / 12$; Signoret, 1996) were observed in the REC1 test (NFB: indiv_9; RELAX: indiv_11, indiv_16, TRT: indiv_23) and in the LEA1 test (TRT: indiv_25).

The analysis of the evolution of the spectral powers of the alpha and thêta frequency bands from session 1 to session 4 highlighted a significant increase of the spectral power of the alpha band, $\mathrm{z}=1.89, \mathrm{p}=.031$ and an increase of the alpha/ thêta relation between the 2 sessions, $\mathrm{z}=2.09, \mathrm{p}=.019$, though no increase of the spectral power of the thêta band was observed, $\mathrm{z}=1.22, \mathrm{p}>.05$. The analysis of individual change (see Table 1) indicated that an increase of the spectral power of the alpha frequency band was observed in 8 participants, that a decrease of the spectral power of the thêta frequency bands was observed in 6 participants and that an increase of the alpha/thêta ratio was observed in 7 participants. These results are indicative

Table 1.

Individual changes between sessions 1 and 4 for alpha and theta frequency band spectral power as well as for alpha/thêta ratio in NFBgroup. The Reliable Change Index (RCI) was computed for every participant in comparison with remainder of the NFB-group participants $\left(^{*}:\right.$ significant change $(p<.05)$ : more important than mean change of remainder of the group).

\begin{tabular}{cccc}
\hline & \multicolumn{3}{c}{ RCI } \\
\cline { 2 - 4 } Participant & alpha & theta & alpha/theta \\
\hline 1 & .045 & $-1.914^{*}$ & 1.206 \\
2 & 1.149 & .423 & 1.286 \\
3 & 1.874 & $-1.778^{*}$ & 1.228 \\
4 & .819 & $1.881^{*}$ & -.112 \\
5 & .737 & .847 & -.074 \\
6 & $2.052^{*}$ & -1.234 & 1.174 \\
7 & .656 & $-2.015^{*}$ & .56 \\
8 & -.891 & $-2.572^{*}$ & $8.578^{*}$ \\
9 & .901 & $-1.653^{*}$ & $2.590^{*}$ \\
10 & -.988 & 1.462 & -.393 \\
\hline
\end{tabular}

of the inter-individual heterogeneity that subtends the changes of EEG activity such as they might be summarized at the level of the NFB group, with certain participants (1, 2, 3, 6, 7 and 9) appearing to be more capable $(4,5,8$ and 10) of modifying their EEG activity than others in the direction encouraged by the training protocol.

Table 2 shows the average performance in the memory tests for each group and at each measurement. Overall an improvement of memory performance was observed in the members of all 3 groups, $\mathrm{F}(2.26)=.376, \mathrm{p}=.000$, eta $^{2}=.624$ [univaried effects: a) REC1 vs. REC2, $\mathrm{F}(1.27)=23.364, \mathrm{p}=.000$, eta $^{2}=.464$; b) LEA1 vs. LEA2, $\mathrm{F}(1.27)=23.351, \mathrm{p}=.000$, $\left.^{2} \mathrm{et}^{2}=.463\right]$. By contrast, no significant effect of group $\mathrm{x}$ memory performance interaction was demonstrated, $\mathrm{F}(4.52)=.720, \mathrm{p}>.05$.

In order to analyze the evolution of memory performance in every member of the NFB group compared with the average memory performance observed in members of the TRT group, we initially confirmed that the hypothesis of equal variances between the two sessions was respected in the TRT group, both for REC, $\mathrm{t}(8)=.138, \mathrm{p}>.05$ and for LEA, $\mathrm{t}(8)=1.620, \mathrm{p}>.05$. Two RCIs - one for REC and one for LEA-were then computed for every member of the NFB group. The ten individual (NFB) vs. group (TRT) comparisons indicated that the memory performance of several members of the NFB group increased significantly more than the average increase of memory performance observed in members of the TRT group. This result was observed in several participants both for REC (indiv_2: $\mathrm{RCI}=2.128, \mathrm{p}=.021 ;$ indiv_5: $\mathrm{RCI}=2.122, \mathrm{p}=.022$, indiv_7: $\mathrm{RCI}=2.935, \mathrm{p}=.003$; unilateral test) and for LEA (indiv_2: $\mathrm{RCI}=2.367, \mathrm{p}=.012 ;$ indiv_6: $\mathrm{RCI}=3.328, \mathrm{p}=.001$; indiv_8: $\mathrm{RCI}=2.367, \mathrm{p}=.012$; unilateral test). However, increased performance in both memory tests was only significant in one member of the NFB group (indiv_2). As for the connections between changes in EEG activity and improved performance in either memory test, we observed no relation between these two variables (phi $=0, p=1.000$ ), nor did we observe, more specifically, any increase of the alpha/thêta ratio in the 5 members of the NFB group who demonstrated a significant increase of their performance in either memory test, $\mathrm{z}=1.753, \mathrm{p}=.063$.

Finally, Figure 2 shows the evolution of the mean tension level during the 4 sessions. The results did not highlight any difference in the initial level of the 3 groups, $F(2.27)=.765$, $p$ $>.05$, although they do point to a significant decrease of the tension level throughout the sessions, $F(3.81)=27.639$, $p$ $=.000$, as well as a significant group $\mathrm{x}$ session interaction effect, $\mathrm{F}(6.81)=3.154, \mathrm{p}=.000$. The post-hoc comparisons helped to account for this interaction effect by the significant decrease of the tension level in the NFB group, $\mathrm{F}(1.27)=45.99, \mathrm{p}=.000$, and the slightly less pronounced decrease in the RELAX group, $\mathrm{F}(1.27)=17.415, \mathrm{p}=.000$, compared with the stable tension level observed in the TRT group, $\mathrm{F}(1.27)=.55, \mathrm{p}>.05$. Finally, the correlation observed between the increase of the alpha/thêta ratio and the decrease of the tension level between sessions 1 and 4 could not be demonstrated for members of the

\section{Table 2.}

Pre- and post-training mean performance (standard deviation in brackets) in the two memory tasks ("Recall" and "Learning") for the $N F B$, relaxation, and control groups.

\begin{tabular}{ccccc}
\hline & \multicolumn{2}{c}{ "Recall” } & \multicolumn{2}{c}{ "Learning" } \\
\cline { 2 - 5 } Group & Session 1 & Session 4 & Session 1 & Session 4 \\
\hline Neurofeedback & $10.00(1.33)$ & $11.10(.99)$ & $10.50(1.08)$ & $11.50(.71)$ \\
Relaxtaion & $9.80(1.48)$ & $10.90(1.20)$ & $10.80(1.14)$ & $11.50(.85)$ \\
Test/retest & $9.80(1.14)$ & $10.40(1.17)$ & $10.40(1.27)$ & $11.10(1.10)$ \\
\hline
\end{tabular}




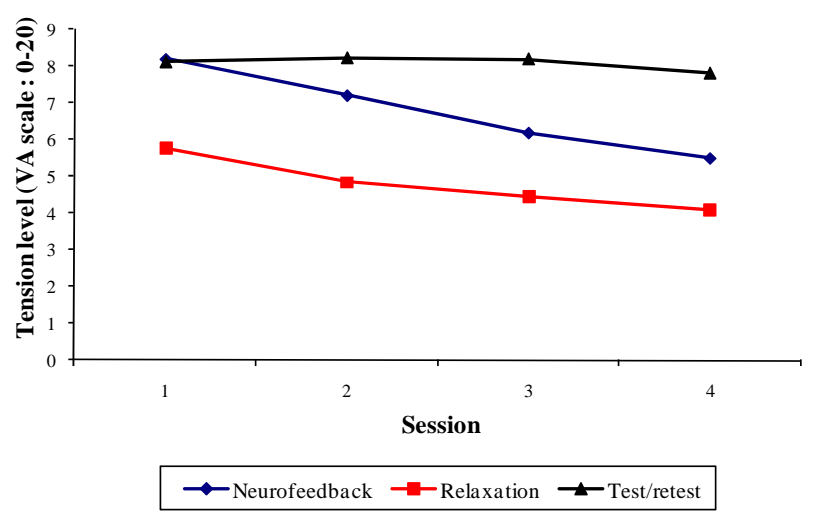

Figure 2.

Mean tension level (stress and anxiety) across the four sessions for the $N F B$-, relaxation- and "waiting-list" groups.

NFB group, $r=.387, \mathrm{p}>.05$.

\section{Discussion}

We have examined the effects of the original training protocol of 4 NFB sessions on memory performance in elderly subjects in good health. The experimental control included 2 control conditions-a so-called relaxation condition and another condition with no particular intervention aiming to assess the test-retest effect on memory performance and tension level.

The first issue was to establish whether the NFB training process that aimed to foster a harmonization of the different ranges of cortical waves in order to tend towards an optimal model of cerebral activity could cause pronounced EEG changes in subjects who had benefitted from it. Considering that memory performance in young adults at rest is related positively to the spectral power of upper alpha waves and related negatively to the spectral power of thêta waves (Hanslmayer et al., 2005; Klimesch, 1999; Klimesch, \& Vogt, 2000), our objective was to train the elderly members of the NFB group to modify both wave ranges in relation to other frequency bands. Analyses showed that NFB training resulted on average in an increase of the spectral power of the upper alpha waves and in an increase of the alpha/thêta ratio. This result needs to be qualified by the fact that the expected changes in EEG activity were not observed in 4 of the 10 members of the NFB group. Our findings corroborate earlier studies carried out on young adults, that had failed to demonstrate any relation between NFB training and 'learned' changes of EEG activity (Egner, \& Gruzelier, 2004; Vernon et al., 2003).

Yet our results indicate that an elderly individual can be trained to modify the amplitude of certain wave ranges and to regulate brain EEG activity more 'efficiently'. Our findings therefore corroborate earlier results pertaining to young adults (Hanslmayer et al., 2005) and elderly adults (Angelakis et al., 2007). In a study of 18 young adults, Hanslmayer and colleagues observed that 5 participants had learned to increase the power of the upper alpha waves, that 6 participants had learned to decrease the power of the thêta waves, that 4 participants had learned to do both and that 3 had failed to learn either (i.e. the non-responders). Angelakis and collaborators (2007) made similar observations in elderly individuals in a study based on a high number of training sessions (between 31 and 36 sessions). They observed that 2 of the 3 members of the experimental group had gradually learned to increase the frequency of peaks of alpha rhythms and that the 3 members of the control group had also learned to increase the amplitude of the alpha waves. It is important to note that in spite of the limited number of training sessions undergone by the subjects involved in the present study, over half of them did learn to increase the spectral power of the upper alpha waves and to reduce the spectral power of the thêta waves.

Once this result had been established, the second issue was to determine whether the NFB used to increase the spectral power of the upper alpha waves and to reduce the spectral power of the thêta waves could lead to an improvement in the cognitive performance of elderly subjects. The alpha waves-particularly the upper alpha waves-have a functional significance in memory processes (Klimesch, 1999; Klimesch et al., 2005), and recent findings suggest that fostering the amplitude of alpha waves could have positive effects on the performance of elderly subjects in a verbal memory task (Angelakis et al., 2007). Contrary to expectations, the observations made in the course of this research do not corroborate this hypothesis. Of course, we observed an improvement in the level of memory performance of NFB group members-albeit an improvement of the same amplitude as the one observed in the 'relaxation' group members and in the 'waiting list' group members.

It appears therefore that this improvement is not indicative of a mere effect of practice. We also established that the EEG activity of elderly subjects in the NFB group whose memory performance improved significantly in comparison with the memory performance of members of the 'waiting list' group had not changed in the way predicted by an effect of NFB. The improved memory performance observed in several members of the NFB group cannot be attributed therefore to changes in the spectral power of upper alpha waves or of thêta waves which NFB training might have enabled. The hypothesis of a relation between NFB training and improved memory performance in elderly subjects is not therefore corroborated by the predicted empirical support.

Envisaged incidentally, the decrease of perceived stress and anxiety in elderly members of the NFB group in the course of the training sessions was a secondary result indicating that in spite of the initial stress generated by the training mechanism, NFB training results in a decrease of the tension level and in an increase of the degree of relaxation in subjects benefitting from such training. In fact, no difference between the tension level of members of the NFB group and the tension level of members of the relaxation group was observed at the end of the four individual sessions. Yet NFB training currently has the status of a 'probably efficient' method for treating anxiety disorders (Moore, 2000). Often conducted with eyes closed and with auditive feedback in patients suffering from anxiety disorders (Hammond, 2006), NFB training is relatively similar to a meditation-based training method that uses sophisticated technology. The NFB training technique used in this research could therefore be useful in the treatment of stress and anxiety in elderly individuals (Ayers, Sorrell, Thorp, Loebach, \& Wetherell, 2007), and, as several recent studies have begun to suggest (Andrealotti, Veratti \& Lachman, 2006; DeLuca, Lenze, Mulsant, Butters, Karp et al., 2005), it may thereby help to reduce or postpone the process of cognitive decline associated with the normal ageing process.

Yet this research does imply several limitations that explain why stimulating alpha waves by NFB training appeared to have no discernable effect on short-term memory in the elderly subjects who took part in this study. Concerning the defined objectives, the number of training sessions undergone by participants is possibly insufficient. While earlier studies have been based 
on 4 or 5 NFB sessions, studies in this research area are often based on approximately ten training sessions (Egner, \& Gruzelier, 2004; Vernon et al., 2003) and the most compelling effects of NFB on cognitive performance were observed when participants were subjected to an even greater number of training sessions (Angelakis et al., 2007). The relative small size of the sample also limits the significance of the results by reducing statistical power and thereby increasing the probability of failing to highlight a statistically significant difference, despite the fact that such a difference did in fact exist. Another limitation of this research is related to the good overall level and relative homogeneity of memory performance displayed by participants, one potential effect of which is a decrease of the chances of observing an improvement of memory performance. Finally, the type of task used in this research to assess memory performance may have been insufficiently matched with the cognitive processes associated with the frequency of wave ranges targeted by NFB training.

\section{Conclusion}

NFB training provides a field of inquiry and potential applications that we are only just beginning to explore in the case of elderly subjects. The present study illustrates this by showing that it is possible to train an elderly subject to modify the amplitude of certain wave ranges according to the optimal model of regulation of EEG activity using an original NFB protocol targeting several wave ranges. Currently, we know that the relations between specific patterns of EEG activity and levels of cognitive performance also require considering NFB as a training technique aimed at encouraging an elderly individual to produce specific patterns of cortical activity in connection with an improved level of cognitive performance. This research avenue, which few have so far ventured down, has tended to generate conflicting results (Bauer, 1976; Angelakis et al., 2997). Our own observations do not corroborate the hypothesis of the effect of NFB training of different frequency ranges (in particular a stimulation of alpha waves) on memory performance in elderly subjects. A more rigorous and more systematic assessment of the effects of NFB training on cognitive performance in elderly subjects is therefore required to improve our understanding of the conditions of application of the different NFB protocols that are applicable to this particular group, to specify the possible indication and to better specify the effects on memory performance.

\section{References}

Albert, A.O., Andrasik, F., Moore, J.L., \& Dunn, B. R. (1998). Thêta/ bêta training for attention, concentration and memory improvement in the geriatric population. Applied Psychophysiology and Biofeedback, 23, 109.

Andrealotti, C., Veratti, B.W., \& Lachman, M.E. (2006). Age differences in the relationship between anxiety and recall. Aging \& Mental Health, 10, 265-271. doi:10.1080/13607860500409773

Angelakis, E., Lubar, J. F., \& Stathopoulou, S. (2004). Electroencephalographic peak alpha frequency correlates of cognitive traits. Neuroscience Letters, 371, 60-63. doi:10.1016/j.neulet.2004.08.041

Angelakis, E., Stathopoulou, S., Frymiare, J. L., Green, D. L., Lubar, J. F., \& Kounios, J. (2007). EEG neurofeedback: A brief overview and an example of peak alpha frequency training for cognitive enhancement in the elderly. The Clinical Neuropsychologist, 2, 110-129. doi:10.1080/13854040600744839

Ayers, C. R., Sorrell, J. T., Thorp, S. R., \& Loebach Wetherell, J. (2007). Evidence-Based Psychological Treatments for Late-Life
Anxiety. Psychology and Aging, 22, 8-17. doi:10.1037/0882-7974.22.1.8

Basar, E. (2004). Memory and brain dynamics: Oscillations integrating attention, perception, and memory. Florida: CRC Press. doi:10.1201/9780203298732

Basar, E., Basar-Eroglu, C., Karakas, S., \& Schürmann, M. (2000). Brain oscillations in perception and memory. International Journal of Psychophysiology, 35, 95-124. doi:10.1016/S0167-8760(99)00047-1

Basar, E., Basar-Eroglu, C., Karakas, S., \& Schürmann, M. (2001). Gamma, alpha, delta and thêta oscillations govern cognitive processes. International Journal of Psychophysiology, 39, 241-248. doi:10.1016/S0167-8760(00)00145-8

Bauer, R. H. (1976). Short-term memory: EEG alpha correlates and the effect of increased alpha. Behavioral Biology, 17, 425-433. doi:10.1016/S0091-6773(76)90793-8

Bearden, T. S., Cassisi, J. E., \& Pineda, M. (2003). Neurofeedback training for a patient with thalamic and cortical infarctions. Applied Psychophysiology and Biofeedback, 28, 241-253. doi:10.1023/A:1024689315563

Brefczynski-Lewis, J. A., Lutz, A., Schaefer, H. S., Levinson, D. B., \& Davidson, R. J. (2007). Neural correlates of attentional expertise in long-term meditation practitioners. Proceedings of the National Academy of Sciences, 104, 11483-11488. doi:10.1073/pnas.0606552104

Budzynski, T. H. (1996). Brain brightening: Can neurofeedback improve cognitive process? Biofeedback, 24, 14-17.

Butnik, S. M. (2005). Neurofeedback in adolescents and adults with attention deficit hyperactivity disorder. Journal of Clinical Psychology, 61, 621-625. doi:10.1002/jclp.20124

Cade, M., \& Coxhead, N. (1979). The Awakened Mind: Biofeedback and the Development of Higher States of Awareness. New York: Delacorte Press.

DeLuca, A. K., Lenze, E. J., Mulsant, B. H., Butters, M. A., Karp, J. F., et al. (2005). Comorbid anxiety disorder in late life depression: association with memory decline over four years. International Journal of Geriatric Psychiatry, 20, 848-854. doi:10.1002/gps.1366

Egner, T., \& Gruzelier, J. H. (2004). EEG biofeedback of low bêta band components: Frequency-specific effects on variables of attention and event-related brain potentials. Clinical Neurophysiology, 115, 131139. doi:10.1016/S1388-2457(03)00353-5

Evans, J. R., \& Abarbanel, A. (1999). Introduction to Quantitative EEG and Neurofeedback. Orlando: Academic Press.

Fernandez, T., Becerra, J., Roca, M., Espino, M., Bahlke, M. Y., Harmony, T., Fernandez-Bouza, A., Belmont, H., \& Diaz-Comas, L., (2008). Neurofeedback in healthy elderly humans with electroencephalographic risk of cognitive impairment. Frontiers in Human Neuroscience. Conference Abstract. doi:10.3389/conf.neuro.09.2009.01.173

Glazer, H. I., \& Laine, C.D. (2006). Pelvic floor muscle biofeedback in the treatment of urinary incontinence: A literature review. Applied Psychophysiology and Biofeedback, 31, 187-201. doi:10.1007/s10484-006-9010-x

Gruzelier, J., Egner, T., \& Vernon, D. (2006). Validating the efficacy of neurofeedback for optimising performance. Progress in Brain Research, 159, 421-431. doi:10.1016/S0079-6123(06)59027-2

Hammond, D. C. (2005). Neurofeedback with anxiety and affective disorders. Child and Adolescent Psychiatric Clinics of North America, 14, 105-123. doi:10.1016/j.chc.2004.07.008

Hammond, D. C. (2006). Neurofeedback treatment of depression and anxiety. Journal of Adult Development, 12, 131-138. doi:10.1007/s10804-005-7029-5

Hanslmayer, S., Sauseng, P., Doppelmayr, M., Schabus, M., \& Klimesch, W. (2005). Increasing individual upper alpha by neurofeedback improves cognitive performance in human subjects. Applied Psychophysiology and Biofeedback, 30, 1-10. doi:10.1007/s10484-005-2169-8

Katsamanis Karavidas, M., Tsai, P. S., Yucha, C., McGrady, A., \& Lehrer, P. M. (2006). Thermal biofeedback for primary Raynaud's phenomenon: A review of the literature. Applied Psychophysiology and Biofeedback, 31, 203-216.

Klimesch, W. (1999). EEG alpha and thêta oscillations reflect cognitive and memory performance: A review and analysis. Brain Research, 
29, 169-195. doi:10.1016/S0165-0173(98)00056-3

Klimesch, W., Vogt, F., \& Doppelmayer, M. (2000). Interindividual differences in alpha and thêta power reflect memory performance. Intelligence, 27, 347-362. doi:10.1016/S0160-2896(99)00027-6

Klimesch, W., Schack, B., \& Sauseng, P. (2005). The functional significance of thêta and upper alpha oscillations for working memory: A review. Experimental Psychology, 52, 99-108. doi:10.1027/1618-3169.52.2.99

Lutz, A., Greischar, L. L., Rawlings, N. B., Ricard, M., \& Davidson, R. J. (2004). Long-term meditators self-induce high-amplitude gamma synchrony during mental practice. Proceedings of the National Academy of Sciences, 101, 16369-16373. doi:10.1073/pnas.0407401101

Markand, O. N. (1990). Alpha rythms. Journal of Clinical Neurophysiology, 7, 163-189. doi:10.1097/00004691-199004000-00003

Maassen, G. H. (2004). The standard error in the Jacobson and Truax Reliable Change Index: The classical approach to the assessment of reliable change. Journal of the International Neuropsychological Society, 10, 888-893. doi:10.1017/S1355617704106097

Masterpasqua, F., \& Healey, K. N. (2003). Neurofeedback in psychological practice. Professional Psychology: Research and Practice, 34, 652-656. doi:10.1037/0735-7028.34.6.652

Middaugh, S. J., \& Pawlick, K. (2002). Biofeedback and behavioral treatment of persistent pain in the older adult: A review and a study. Applied Psychophysiology and Biofeedback, 27, 185-202. doi:10.1023/A:1016208128254

Moore, N. C. (2000). A review of EEG biofeedback treatment of anxiety disorders. Clinical Electroencephalography, 31, 1-6.

Nestoriuc, Y., \& Martin, A. (2007). Efficacy of biofeedback for migraine: A meta-analysis. Pain, 128, 111-127. doi:10.1016/j.pain.2006.09.007

Obrist, W. D. (1954). The electroencephalogram of normal aged adults. EEG Clinical Neurophysiology, 6, 235-244. doi.org/10.1016/0013-4694(54)90025-5

Sauseng, P., \& Klimesch, W. (2008). What does phase information of oscillatory brain activity tell us about cognitive processes. Neuroscience Biobehavioral Research, 32, 1001-1013. doi.org/10.1016/j.neubiorev.2008.03.014

Scott, W. C., Kaiser, D., Othmer, S., \& Sideroff, S. I. (2005). Effects of an EEG biofeedback protocol on a mixed substance abusing population. The American Journal of Drug and Alcohol Abuse, 31, 455-469. doi.org/10.1081/ADA-200056807

Signoret, J.-L. (1996). B.E.C. 96: Evaluation des troubles de mémoire et des désordres cognitifs associés. Paris: Ipsen.

Sterman, M. B., \& Egner, T. (2006). Foundation and practice of neurofeedback for the treatment of epilepsy. Applied Psychophysiology and Biofeedback, 31, 21-35. doi.org/10.1007/s10484-006-9002-x

Vernon, D. J. (2005). Can neurofeedback training enhance performance? An evaluation of the evidence with implications for future research. Applied Psychophysiology and Biofeedback, 30, 347-364. doi.org/10.1007/s10484-005-8421-4

Vernon, D., Egner, T., Cooper, N., Compton, T., Neilands, C., Sheri, A., \& Gruzelier, J. (2003). The effect of training distinct neurofeedback protocols on aspects of cognitive performance. International Journal of Psychophysiology, 47, 75-85 doi.org/10.1016/S0167-8760(02)00091-0

von Stein, A., Chiang, C., \& Konig, P. (2000). Top-down processing mediated by interarea-synchronization. Proceedings of the National Academy of Sciences, 26, 14748-14753. doi.org/10.1073/pnas.97.26.14748 